\title{
EFFECTIVENESS OF IMMUNO-CHROMATOGRAPHIC TEST IN THE DIAGNOSIS OF FALCIPARUM MALARIA
}

\author{
Uddin $\mathrm{MM}^{1}$, Hussain $\mathrm{SMB}^{2}$, Rahman $\mathrm{MM}^{3}$, Hossain $\mathrm{MD}^{4}$
}

\begin{abstract}
Introduction : Various diagnostic tools are used to confirm diagnosis of different types of malaria. Immunochromatographic test for Plasmodium falciparum (ICT malaria Pf) is one of them.

Objective: Aim of this prospective study was to find out a rapid, effective and easy tool for the diagnosis of malaria for the hilly area of Bangladesh.

Method: A prospective study was conducted at hilly area of Bangladesh over a period of 7 months. Clinically suspected 50 cases of malaria of both sexes of different age group were included in the study. Blood samples were collected and simultaneously microscopic examination of Leishman's stain film and ICT malaria Pf were done. Results were compared using standard method.

Result: The mean age of the patients was $29.9 \pm 18$ years and 49 were male. Among the 19 parasitaemic cases, $16(84.2 \%)$ cases were Plasmodium falciparum positive, $02(10.5 \%)$ cases were Plasmodium vivax positive and $01(5.3 \%)$ was mixed infection. The degree of parasitaemia (malarial parasite count /cmm) ranges form $2000 / \mathrm{cmm}$ to $30,000 / \mathrm{cmm}$ with the mean $10,395 \pm 8,444 / \mathrm{cmm}$. Among the 19 microscopically positive cases, $16(84.2 \%)$ cases were ICT positive and $03(15.8 \%)$ cases were ICT negative. The sensitivity of ICT for Pf in this study was $94.1 \%$ and the specificity of ICT for Pf was $100 \%$. The positive predictive value (PPV) and negative predictive value (NPV) were $100 \%$ and $97.1 \%$ respectively
\end{abstract}

Conclusion: The study revealed that the "ICT Malaria Pf has a very important role in the diagnosis of Plasmodium malaria. The sensitivity and specificity are approx $100 \%$ and very rapid in action. Key words: Falciparum malaria, Immunochromatographic test, Hilly area of Bangladesh

\section{Introduction}

Malaria is one of the most common parasitic diseases and a major health problem world wide infecting 200 million and killing about 02 million people each year ${ }^{1}$.
Despite the problem of drug resistance, malaria is a curable disease if patient have access to early diagnosis and prompt treatment ${ }^{2}$.

Rapid and accurate diagnosis is the key to effective disease management and one of the basic technical elements of the strategy for malaria control ${ }^{3}$. So far the light microscopic examination (ME) of the stained thick and thin blood film is the standard method of malaria diagnosis ${ }^{4,5}$. ME however, requires well trained and experienced malaria microscopist and is also rather time consuming. Some other techniques such a quantitative buffy coat (QBC) and polymerase chain reaction (PCR) need expensive equipment and material as well as have neither been adapted nor are feasible for the malaria field works in the endemic area. Antibody detection by serologic tests usually shows prior exposure rather than current infection ${ }^{6}$, particularly in the residents of malaria endemic area.

Therefore the recent introduction of rapid diagnostic tests for malaria is of considerable interest. Such tests are based on the detection of antigen released from parasitized red cells. The specific antigens for Plasmodium falciparum ( $P$ falciparum) is histidine-rich protein (Pf HRP-2) and plasmodium lactate dehydrogenase $(\mathrm{pLDH})$ for all other human malaria parasites ${ }^{7}$. These are soluble antigens released by the parasites and are detectable in the blood samples taken from parasitaemic malaria patients. Detection of pf HRP-2 and pLDH antigens are performed by IgG monoclonal antibodies, which are prepared against these antigens.

The main objective of this study is to evaluate the immunochromatographic tests for $P$ falciparum (ICT malaria Pf) in the detection of parasitaemia in falciparum malaria infection on the back ground of Chittagong Hill Tracts, the malaria endemic area of Bangladesh.

\section{Materials and Methods}

This was a prospective study carried out at $\mathrm{CMH}$ Chittagong from April 2009 to October 2009. Fifty patients from both sexes and all age groups with clinical

1. Lt Col Mohammad Mosleh Uddin, MBBS, DCP, MCPS, FCPS, Classified Specialist in Pathology, CMH Chittagong; 2. Brig Gen Sk Md Bahar Hussain, MBBS, FCPS, FRP(E), FRCP(G), FACP, Adviser Specialist in Medicine and Senior Physician, CMH Chittagong; 3. Lt Col Md Mizanur Rahman, MBBS, DCP, MCPS, FCPS, Classified Specialist in Pathology, CMH Savar; 4. Lt Col Md Delwar Hossain, MBBS, FCPS(Med), FCPS (Gastro), Graded Specialist in Medicine, CMH Chittagong. 
suspicion of malaria having history of fever (temperature above $99^{\circ} \mathrm{F}$ ) at the time of presentation associated with shivering in some case and with other nonspecific symptoms like headache, body ache and fatigue were included in the study.

Venous blood was collected by the standard vene-puncture procedure into ethylene diamine tetraacetate acid (EDTA) tube for microscopy and immunochromatographic testing. Thick and thin films were prepared and stained with Leishman's and Giemsa stain and examined under microscope. Minimum 200 consecutive fields were counted in thick blood film before classifying result as negative. Fifteen microlitre whole blood was applied to a sample pad impregnated with colloidal gold labelled antibodies directed against HRP-2 antigen. The result could then be read 5 minutes after the colour of blood has almost cleared. The test was considered valid if the control line was visible and positive if the test line was visible. The test was considered negative, when only a control line was visible. In case that no line or only the test line appeared, the result was considered to be invalid. Thin films and test strips were read blind to each other.

Data was collected and analyzed using Epi info (Version6). For sensitivity and specificity, the ICT malaria test results were compared with microscopy using as a gold standard. Variable measures were the number of true positives (TP, means test correctly identifies the patient with disease), number of true negatives ( $\mathrm{TN}$, means test is negative and person is without disease), number of false positives (FP, means test is positive in a patient without disease) and number of false negatives (FN, means test is negative in a patient with disease). Sensitivity was calculated as TP/TP+FN, specificity was calculated as TN $/ \mathrm{TN}+\mathrm{FP}$, the positive predictive value (PPV) was calculated as $\mathrm{TP} / \mathrm{TP}+\mathrm{FP}$ and negative predictive value (NPV) was calculated as TN/TN+FN as suggested by Tijra et $\mathrm{al}^{11}$.

\section{Result}

The mean age of the patients in this study was $29.9 \pm 18$ years and the range was $18-56$ years. Among the patients under this study, 49 were male and only 01 was female. The total number of patients with Plasmodium parasitaemia was $19(38 \%)$ and patients without parasitaemia were 31 $(62 \%)$. The detail is shown in (Table-I).

Table-I: Distribution of Patient as per parasitaemic state $(\mathrm{n}=50)$

\begin{tabular}{|c|l|}
\hline State & Number (\%) \\
\hline Parasitaemic & $19(38)$ \\
\hline Plasmodium falciparum & $16(84.2)$ \\
Plasmodium vivex & $02(10.5)$ \\
Mixed Plasmodium & $01(05.3)$ \\
\hline Non - parasitaemic & $31(62)$ \\
\hline
\end{tabular}

The degree of parasitaemia (malarial parasite count $/ \mathrm{cmm}$ ) varies among the positive cases. The range was $2000 / \mathrm{cmm}$ to $30,000 / \mathrm{cmm}$ and the mean $10,395 \pm 8,444 / \mathrm{cmm}$. The total number of positive cases by Immunochromatographic test (ICT) for $P$ falciparum was $16(84.2 \%)$ among the total 19 microscopically positive cases (table -I). Those 16 cases were positive both microscopically and by ICT for Pf. Other 3 microscopically positive cases were ICT negative. One case was mixed which was negative by ICT for Pf. The sensitivity of ICT for Pf in this study was $94.2 \%$. Among the total 50 cases, 31 cases were negative by both microscopically and by ICT for Pf.

Table -II: Performance Characteristics of ICT for Pf

\begin{tabular}{|l|l|}
\hline Performance & Level \\
\hline Sensitivity & $94.2 \%$ \\
\hline Specificity & $100 \%$ \\
\hline PPV & $100 \%$ \\
\hline NPV & $97.1 \%$ \\
\hline
\end{tabular}

\section{Discussion}

The recommended method and current gold standard used for the routine laboratory diagnosis of malaria is the microscopic examination of stained thick and thin blood films. In capable hands, this method can be expected to detect 50 parasites/cmm $(0.001 \%)$ parasitaemia and to identify to the species level $98 \%$ of all parasites seen. However, this procedure is difficult and time consuming, and requires skilled staff ${ }^{8}$.

In the past few years efforts have been made to replace the reading of blood film by other techniques. Fluorescence and PCR have proven to be sensitive but difficult to perform in a routine laboratory practice ${ }^{9}$. Therefore, the development of easy, rapid and accurate tests for the detection of plasmodia infection is highly desirable ${ }^{10}$. Immunochromatographic rapid tests offer the possibility of more rapid non-microscopic method for rapid diagnosis ${ }^{9,10}$. In this study the performance of ICT malaria Pf test with traditional light microscopy was being investigated. The study showed $94.1 \%$ sensitivity and $100 \%$ specificity for $P$ falciparum with a PPV and an NPV of $100 \%$ and $97.1 \%$ respectively.

Various studies using monoclonal antibody to HRP-2, same as in present study, revealed comparable results in terms of sensitivity (range $92-100 \%$ ) and specificity (range 84 $99 \%$ ) done at international levels ${ }^{11,-14}$. Similar results were reported by Gasser et $\mathrm{al}^{15}$ using the same kit. However, a study from Indonesia reported a low sensitivity for HRP-2 antigen detection tests ${ }^{16}$. For the detection of $P$ falciparum, the sensitivity and specificity of ICT Pf / Pv (current study), ICT Malaria Pf ${ }^{13-17}$ Parasight $F^{18,19}$ for HRP-2 were at least equal to those of microscopy performed in a wellorganized malaria diagnostic laboratory and much better than those routinely achieved in remote primary health centres $^{20,21}$. 
In this study one subject with mixed infection was not detected by HRP-2 antigen. This may be due to low level of parasitaemia, very high levels of parasitaemia or gene deletion for the production of HRP-2 antigen. These findings have also been reported in previous studies ${ }^{11,18-20}$. However the sensitivity of all rapid immunochromatographic tests is low when compared with $\mathrm{PCR}^{22}$.

The dipstick test should not replace the need for blood film examination, as it is not $100 \%$ sensitive at low parasitaemia and repeated daily testing may be necessary to establish the diagnosis. Nor does the test give any indication of density of parasites, essential in planning of management.

\section{Conclusion}

At present for the diagnosis of malaria in endemic areas, the rapid diagnostic test kits are rather expensive ${ }^{6}$ as compared to microscopic examination. Nevertheless, it is very helpful for the remote areas as well as for clinics and health centres where necessary facilities for ME are not accessible for the prompt diagnosis of falciparum infection, particularly in patients with severe and complicated malaria.

\section{Refernces}

1. Cattani J, Davidson D, Enger H. Malaria in Tropical disease research Progress 91-92. 11th Programme Report of the UNDR World Bank, WHO, Geneva World Health Organization; 1993:15-27.

2. World Health Organization. A rapid dipstick antigen capture assay for the diagnosis of falciparum malaria. Bull of World Health Organization 1996; 47-54.

3. World Health Organization. A global strategy for malaria control. Geneva: World Health Organization; 1997.

4. Iqbal J, Khalid N, Hira PR. Comparison of two commercial assays with expert microscopy for confirmation of symptomatically diagnosed malaria . J Clin Microbial 2002; 40:4675-8.

5. Afzal S, Zia N, Fazal M, Ahmed M, Mubarik A, Qureshi AH. Malaria- an experience at CMH Badin (Sindh). Pak J Pathol 2003; 14:16-20.

6. World Health Organization. Malaria diagnosis : new perspectives. Geneva: World Health Organization; 2000:14.

7. Saxena N, Moody A. Rapid diagnostic tests for malaria parasites. Clin Microbiol Rev 2002; 15: 66-78.
8. Craig MH, Sharp BL. Comparative evaluation of four techniques for the diagnosis of Plasmodium falciparum infections. Trans R Soc Trop Med Hyg 1997;91:279 - 282.

9. Harani SM, Beg AM, Khaleeq L, Adil NS, Kakepoto NG, Khurshid M. Role of ICT Malaria Immunochromatographic Test for Rapid diagnosis of malaria. J Pak Med Assoc 2006; 56: 167 -171.

10. World Health Organization. Malaria Rapid Diagnostic test performance results: Round 1 (2008). Geneva: World Health Organization; 2009.

11. Tjitra E, Suprianto S, Dyer M, Currie BJ, Anstey NM. Field evaluation of the ICT malaria p.f/p.v immunochromatographic test for detection of Plasmodium falciparum and Plasmodium vivax in patients with a presumptive clinical diagnosis of malaria in Eastern Indonesia. J Clin Microbiol 1999; 37: 2412 -7.

12. Rahim F, Haque AU, Jamal S. Comparison of Amrad ICT test with microscopic examination for rapid diagnosis of malaria. J Coll Physicians Pak 2002; 12: 530 -3.

13. Kilian AH, Mughusu EB, Kabagambe A, von Sonnenberg F. Comparison of two rapid HER2 -based diagnostic tests for Plasmodium falciparum. Trans R Soc Trop Med Hyg 1997; 91: 666-7.

14. Singh N, Saxena A, Valecha N.Field evaluation of ICT Malaria P.f/P.v immunochromatograph test for diagnosis of Plasmodium falciparum and P. vivax infection in forest villagers of Chhandwara. Central India. Trop Med Int Health 2000; 5: 765 - 70.

15. Gasser RA Jr, Arevalo I, Miller RS, Magill AJ, Forney JR, Sirichaisinthrop J et al. Primary evaluation of the NOW ICT malaria P.f/P.v rapid diagnostic device for the detection of Plasmodium falciparum and Plasmodium vivax (abstract). Am J Trop Med Hyg 2001; 65 (Suppl):320.

16. Durrheim DN, Ia Grange JJ, Govere J, Mngomezulu NM. Accuracy of a rapid Immunochromatographic card test for Plasmodium falciparum in a malaria control programme in South Africa. Trans R Soc Trop Med Hyg 1998; 92: 32 -3.

17. Ende VJ, Vervoot T, van Gompel A, Lynen L. Evaluation of two tests based on the detection of histidine rich protein-2 for the diagnosis of imported Plasmodium falciparum malaria. Trans R Soc Trop Med Hyg 1998; 92 : $285-8$.

18. World Health Organization. A rapid dipstick antigen assay for the diagnosis of falciparum malaria. WHO informal consultation on recent advances on diagnostic techniques and vaccines for malaria Bull. WHO 1996; $74: 47$-54.

19. Moody A. Rapid diagnostic tests for malaria parasites. Clin Microbiol Rev 2002; 15: 66 - 78

20. Pieroni P, Mills CD, Ohrt C, Harrington A, Kain KC. Comparison of the Parasight - F and ICT malaria P.f test with the polymerase chain reaction for the diagnosis of Plasmodium falciparum in travellers. Trans R Soc Trp Med Hyg 1998; 92: 166 -9.

21. Lubell Y, Reyburn H, Mbakilwa H, Mwangi R, Chonya K, Whitty CJ, Mills A. The impact of response to the results of diagnostic tests for malaria, cost -benefit analysis. BMJ 2008; 336(7637): 202-205.

22. Iqbal J, Sher A, Hira PR, Al-Owaish R. Comparison of the OptiMAL test with PCR for the diagnosis of malaria in immigrants. J Clin Microbiol 1999; 37: 3644 - 6. 